\title{
Ueber eine neue Methode der Bestimmung des Schwefels in unorganischen Sulfiden;
}

\author{
von \\ Paul Jannasch.
}

Im Anschluss an eine frühere Abhandlung ${ }^{1}$ ) über eine neue Methode der Pyrit-Analyse machte ich bereits eine nachträgliche Angabe über die energische Wirkung des Sauerstoffs auf erhitztes Pyritpulver, aus welcher Thatsache sich eine sehr einfache und rasch zum Ziele führende Methode, das genannte Mineral zu analysiren, ergab.

Ich habe mich inzwischen bemüht, der leichten Oxydirbarkeit unorganischer Sulfide in einem Sauerstoffstrome eine allgemeine analytische Anwendung zu verleihen, und aus diesem Grunde meine quantitativen Versuche auch auf andere Sulfide wie Pyrit ausgedehnt, und zwar mit bestem Erfolge.

Als Absorptionsgefässe für die gebildete Schwefelsäure benutzte ich damals eine zweifach tubulirte Vorlage in Verbindung mit einer Péligot'schen Röhre und einem offnen Cylinder, ein Apparat, wozu man 4 durchbohrte Korke braucht, in deren Poren nur allzu leicht kleine Säuremengen bei dem Abspritzen zurückbleiben und so der Analyse verloren gehen. Um disem Uebelstande abzuhelfen, bediene ich mich gegenwärtig des folgenden, mit Ausnahme eines Korkes und eines Kautschukschlauchendes, nur aus Glastheilen bestehenden Apparates $\left.{ }^{2}\right)$, dessen Einrichtung die beigegebene Zeichnung klar und deutlich zeigen soll.

Die von mir benutzten Absorptionscylinder besitzen eine Höhe (excl. Röhreneinsatz) von $203 \mathrm{Mm}$. bei einer Innenweite von $36 \mathrm{Mm}$. und einem Fassungsraum von ca. $170 \mathrm{Ccm}$. Der senkrecht stehende Vorstoss $d$ ist $58 \mathrm{Mm}$. hoch bei $15 \mathrm{Mm}$. Weite im Lichten und wird vermittelst eines gesunden Korkes mit dem ausgezogenen und rechtwinklig umgebogenen Ver-

1) Dies. Journ. [2] 40, 233.

2) Derselbe wird von der hiesigen mechanischen Werkstätte von C. Desaga (Inhaber: A. Rodrian u. G. Beck) geliefert. 
Bestimmung des Schwefels in unorganischen Sulfiden. 567

brennungsrohr verbunden, durch welch letztere Anordnung sich am allersichersten ein Sichlockern der Glasverschlüsse während des Verlaufes der Analyse verhüten lässt. Cylinder $a$ und $b$ sind durch ein Endchen Gummischlauch dicht aneinander liegend vereinigt. Der am Ende noch vorgelegte ErlenmeyerKolben fasst ca. $600 \mathrm{Ccm}$. bei einer Höhe von $173 \mathrm{Mm}$. Die Menge des Bromwassers rechne ich für je einen Cylinder auf 60-70 Ccm., während in der letzten Vorlage $c$ das Entwicklungsrohr nur wenig einzutauchen braucht. $f$ ist das Porzellanschiffchen mit der Substanz.

Zum Gelingen der Analyse sind die folgenden Bedingungen unerlässlich. Das Verbrennungsrohr ${ }^{1}$ ) darf nicht zu eng

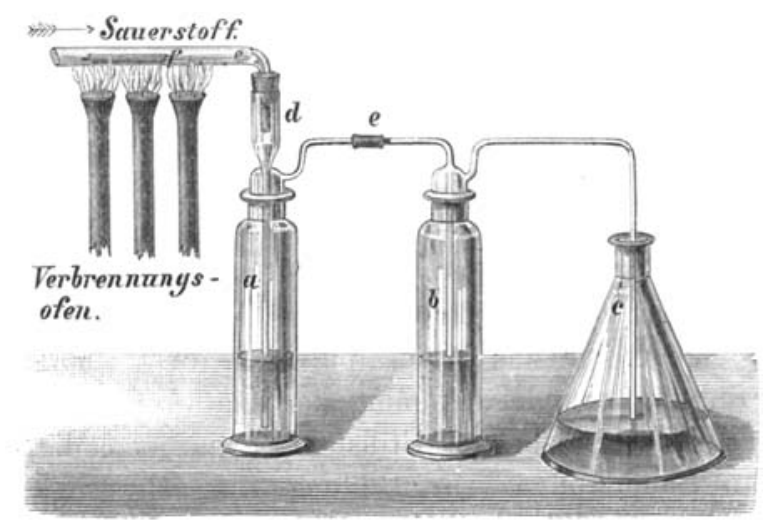

sein, es muss wenigstens $15 \mathrm{Mm}$. Weite im Lichten besitzen, so dass es ein geräumiges Porzellanschiffchen fasst, worin die Substanz in dünner Schicht ausgebreitet wird. Das zu analysirende Sulfidpulver ist möglichst fein gepulvert und in nicht zu reichlicher Menge (nicht über 0,6 Grm.) zu verwenden. Die Schnelligkeit des Sauerstoffstromes regulire man auf 150-200 mittelgrosse Blasen pro Minute, und zwar nehme man den Gasstrom im Anfang nicht zu langsam, um einem Rückwärtsdruck der gebildeten schwefligen Säure vorzubeugen; ist das freiwillig eintretende Verglimmen der Substanz vorüber, so wird man nun den Sauerstoffstrom zweckentsprechend verlangsamen. Zunächst zündet man nur die hinterste Flamme unter

1) Das von mir jetzt benutzte Verbrennungsrohr hatte eine Länge von $60 \mathrm{Cm}$. 
dem Schiffchen und allenfalls auch noch die darauf folgende an und wartet das Eintreten des Selbsterglühens ab, welches sich alsdann durch die ganze Masse weiter verbreitet. Je nach der Lebhaftigkeit der Reaction und den zu beobachtenden Absorptionserscheinungen hat man mit dem Weiterglühen fortzufahren und dasselbe zu vollenden, resp. den Sauerstoffstrom zu mässigen u. s. f. Für den Fall eines gänzlichen zeitweiligen Abschlusses des letzteren, ohne gleichzeitigen Abschluss des Gasometerhahnes, dient ein zwischen den Rohr und dem Trocken-Apparat eingeschalteter Quetschhahn. Der vor dem Schiffchen liegende Röhrentheil $e$ wird von Anfang an schwach erwärmt zum unmittelbaren Uebertreiben der als Nebenprodukt entstehenden Schwefelsäure. Nachdem nun die Substanz bei vorliegenden Kacheln vollkommen ins Glühen gekommen, muss die Einwirkung des Sauerstoffs wenigstens noch eine halbe Stunde andauern, erforderlichen Falls sogar dreiviertel Stunden (cf. weiter unten bei Zinkblende), um einerseits allen Schwefel zu oxydiren und andererseits auch die letzten Spuren von Schwefelsäure aus etwa gebildetem Metallsulfat auszutreiben, was bei Anwendung kräftiger Glühhitze mit Sicherheit erreicht wird. So lange sich das Mineralpulver in dem Zustande der Selbsterglühung befindet, kommt der Gasstrom in den Absorptionscylindern fast zum Stillstande, da jetzt die Hauptmenge an Sauerstoffgas zur Bildung von schwefliger Säure verbraucht wird. Entsteht bei der Oxydation des vorliegenden Sulfids ausschliesslich schweflige Säure, wie beispielsweise bei der Zinkblende, so entfärbt sich die Flüssigkeit im ersten Cylinder vollständig, es ist deshalb gerathen, derselben für alle Fälle einige Tropfen Brom besonders hinzuzufügen. Ist die vollständige Oxydation der Substanz vollendet und der Sauerstoffstrom unterbrochen, so wartet man mit der Auseinandernahme des Apparates und dem sorgfältigen Ueberspülen der Schwefelsäurelösung in ein grösseres Becherglas, bis aller in den zwei Cylindern angesammelte Qualm von Schwefelsäure absorbirt ist und die Gefässe völlig klar erscheinen. Während dieser Zeit einen ganz langsamen Sauer-, eventuell Luftstrom durch den Apparat streichen zu lassen, kann dem gewünschten Zwecke nur förderlich sein. Zu der im Becherglas befindlichen Gesammtlösung wird nun $1 \mathrm{Ccm}$. concentrirte Salzsäure gesetzt 
und die Flüssigkeit auf der Asbestschale, oder auf einem mit Asbestpapier überdeckten Drahtnotz so lange verdampft, bis alles Brom entwichen ist und die Lösung völlig farblos aussieht. Das vollständige Verjagen des freien Broms erscheint absolut geboten, insofern es die Baryumsulfatfällung benachtheiligt, wohl in Folge von Löslichkeitsverhältnissen, ein Verhalten, woran die gleichzeitig vorhandene Bromwasserstoffsäure ebenfalls betheiligt sein kann. Handelt es sich deshalb um äusserste Genauigkeit und drängen nicht die Zeitumstände, so verdampft man alles $W$ asser in einer Porzellanschale und nimmt schliesslich die Fällung in einer rein salzsauren Lösung vor. Das Brom durch Zusatz von etwas Alkohol während des Verdampfens möglichst rasch zu entfernen, wurde aufgegeben, als sich herausstellte, dass dadurch der SchwefelGehalt zu niedrig ausfiel. Es hatten sich also hierbei offenbar geringe Mengen von Aethylsulfosäure gebildet.

Bei der Fällung der reichlichen Quantität Schwefelsäure durch Baryumchlorid ist vor allen Dingen jeder Ueberschuss des letzteren thunlichst zu vermeiden, welchen $Z_{w e c k}$ man am allereinfachsten erreicht, wenn man sich einer zehnprocentigen Baryumchloridlösung bedient (vgl. Lunge's Taschenb. f. Sodafabrik. etc. S. 91) und für jeden speciellen Fall die erforderliche Cubikcentimeteranzahl derselben annähernd berechnet. Durch mehrmaliges Decantiren des Niederschlags mit reichlichen Mengen von kochendem Wasser (auch bei Salzsäurezusatz) lässt sich das durch eine unvorsichtige Fällung in den Niederschlag mit eingegangene Baryumchlorid nicht völlig entfernen, und das mehrmalige Extrahiren des geglühten Baryumsulfats mit salzsaurem Wasser ist schon deshalb ausgeschlossen, weil dadurch gleichzeitig das durch die reducirende Wirkung der Filterkohle etwa entstandene Baryumoxyd oder Baryumsulfid mit verloren geht. Niemals aber darf das geglühte Baryumsulfat wiederholt mit einer schwachen Salzsäure behandelt werden, weil dieses Verfahren ganz directe Verluste an Baryumsulfat im Gefolge hat.

Das Gesammitfiltrat von dem ausgefällten Baryumsulfat, welches häufig mehr als einen Liter beträgt, muss unter Umständen eingedampft werden; ich habe gar nicht selten hierbei die Ausscheidung von gelöst gewesenem Baryumsulfat be- 
obachtet. Alle diejenigen Flüssigkeiten, in denen vor der Fällung mit Baryumchlorid noch ein wenig Brom vorhanden war, zeigten dieses Verhalten in hohem Grade, so dass die betreffenden Nachfällungen mitunter 0,005-0,020 Grm. an Barymsulfat betrugen.

\section{Analyse von Zinkblende.}

Die Zinkblende wurde wider Erwarten schwierig von Sauerstoffgas angegriffen und eine vollständige Oxydation nur durch starkes und anhaltendes Glühen erzielt, daher es sich ereignete, dass die ersten Analysen, zu welchen das Mineral nicht fein genug gepulvert genommen war, ein $z u$ niedriges Resultat lieferten 1). Bedingung zum Gelingen der Analyse ist die Anwendung von sammtweich sich anfühlendem Pulver und nach dem Verglimmen der Blende ein nachträgliches dreiviertelstündiges, möglichst kräftiges Glühen der Substanz. Das Selbsterglühen des Pulvers erfolgt erst nach 10-15 Minuten Glühtemperatur unter ausschliesslicher Bildung von schwefliger Säure.

Das zuïckbleibende Zinkoxyd (natürlich nur von ganz reinen, eisenfreien Blenden) kann behufs Zinkbestimmung direct im offenen Schiffchen gewogen werden, oder man schiebt letzteres zu diesem Zweck sammt Inhalt in ein verschliessbares Wägerohr. Nach Behandlung mit Salzsäure und Ausglühen wird später das Schiffchen zurückgewogen. Das Präparat von Zinkoxyd soll sich klar in verdünnter kalter Salzsäure lösen, ohne hierbei Geruch nach Schwefelwasserstoff zu entwickeln. Das aus der Lösung mit Natriumcarbonat gefällte und gewogene Zink liefert durchschnittlich um ein Geringes höhere Werthe.

a) Analyse einer durchsichtigen, hellgelben spanischen Zinkblende rom Picos de Europa.

1. $0,4762 \mathrm{Grm}$. Blende gaben $0,3965 \mathrm{Grm} . \mathrm{ZnO}=0,3182 \mathrm{Grm} . \mathrm{Zn}$; 1,1372 Grm. $\mathrm{BaSO}_{4}=0,1563$ Grm. S und 0,00024 Gangart.

2. $0,5720 \mathrm{Grm}$. Substanz gaben $1,3708 \mathrm{Grm}$. BaSO $4=0,1884 \mathrm{Grm}$. S.

3. $0,5350 \mathrm{Grm}$. Substanz gaben $1,2747 \mathrm{Grm}$. $\mathrm{BaSO}_{4}=0,1752 \mathrm{Grm}$. S.

1) Bemerkt sei hier, dass sich bei dem Pulvern der Zinkblende regelmässig ein schwacher, aber unzweifelhafter Geruch nach Schwefelwasserstoff bemerkbar macht. 
Bestimmung des Schwefels in unorganischen Sulfiden. 571

4. 0,6032 Grm. Substanz lieferten, mit Königswasser und Brom aufgeschlossen $=1,4525$ Grm. $\mathrm{BaSO}_{4}=0,1996 \mathrm{Grm}$. S.

5. 0,5572 Grm. Substanz in gleicher Weise behandelt, gaben 1,3324 Grm. $\mathrm{BaSO}_{4}=0,1831$ Grm. S.

Somit lieferte die Aufschliessung der Zinkblende im Sauerstofistrome:

$\begin{array}{rrcc}\text { Zn } & 1 . & 2 . & 3 . \\ \text { S } & 32,82 \% & - & - \\ \text { Gangart } & 0,05 \% & 32,94 \% & 32,75 \% \\ & -99,69 \% & - & -\end{array}$

und die gewöhnliche Methode auf nassem Wege:

$$
\begin{aligned}
& 4 . \quad 5 \text {. } \\
& 33,09 \% \mathrm{~S} \quad 32,86 \% \mathrm{~S} .
\end{aligned}
$$

b) Analyse einer schwarzen englischen Zinkblende von Alston in Cumberland.

Die folgenden, mit grösster Sorgfalt ausgeführten Zinkblendeanalysen verdanke ich meinem Schüler, Herrn stud. Karl Hefft (aus Heidelberg).

1. Analyse durch Oxydation der Blende im Sauerstoffstrome. $0,5243 \mathrm{Gr}$. Substanz lieferten 1,3550 Grm. BaSO $=0,1862 \mathrm{Grm} . \mathrm{S}$; 0,8529 Grm. $\mathrm{ZnO}=0,2832 \mathrm{Grm}$. Zn; 0,0742 Grm. $\mathrm{Fe}_{2} \mathrm{O}_{3}=0,05194 \mathrm{Grm}$. Fc; 0,0011 Grm. Gangart und Spuren von Bleisulfat.

2. Analyse auf nassem Wege. Hierzu wurde die fein zerriebene Blende im Bunsen'schen grossen Porzellantiegel mit Königswasser aufgeschlossen, unter Zuhülfenahme von Brom zur völligen Oxydation des ausgeschiedenen Schwefels. Die Schwefelsäure bestimmte Herr Hefft in einer besonderen Portion und entfernte vorher das Eisen aus der heissen Lösung mit einem mässigen Ueberschuss von Ammoniak ${ }^{1}$ ), wobei nur der grössere Theil des Zinks gelöst bleibt, welches Metall der Baryumsulfatfällung weniger schadet. Das Baryumsulfat wurde dreimal mit salzsaurem, kochendem Wasser decantirt, um es frei von Zinksalz zu erhalten. Die quantitative Bestimmung des Zinks neben dem Eisen erfolgte durch Natriumacetat, wobei hauptsächlich dafür Sorge zu tragen ist, dass man die in der ursprünglichen Lösung vorhandene freie Salzsäure mit Soda nicht zu weit neutralisirt, also nicht bis zum Auftreten einer bleibenden braunrothen Färbung, vielmehr danach tropfenweise so viel Salzsäure hinzufügt, bis die klare

1) Dies. Journ. [2] 39, 321; 40, 236 u. 239. 
Flüssigkeit wieder eine rein hellgelbe Farbe angenommen hat. Hierauf kocht man einige Minuten mit dem überschüssigen Natriumacetat, lässt den Niederschlag sich absetzen, filtrirt und wäscht mit kochendem Wasser aus. Verfährt man auf diese Weise, so hat man nicht nöthig, das basische Eisenacetat wieder zu lisen und die Fiallung zur Gewinnung eines zinkfreien Niederschlages noch einmal vorzunelmen. Das Zink kann jetzt aus dem Filtrat entweder mit Natriumcarbonat in der Kochhitze, oder als Zinksulfür mit Schwefelwasserstoffgas gefälltt werden.

Resultate der Analyse.

1. $0,4038 \mathrm{Grm}$. Zinkblende ergaben $1,0425 \mathrm{Grm} . \mathrm{BaSO}_{4}=0,1433 \mathrm{Grm}$. $S$ und 0,0005 Grm. Gangart.

2. $0,8444 \mathrm{Grm}$. Substanz lieferten $0,1244 \mathrm{Grm}$. $\mathrm{Fe}_{2} \mathrm{O}_{3}=0,08708 \mathrm{Grm}$. Fe; 0,5692 Grm. $\mathrm{ZnO}=0,4568 \mathrm{Grm}$. $\mathrm{Zn}$ und Spuren von Bleisulfat.

\begin{tabular}{lcc} 
& 1. (Sauerstoff-Methode) & 2. A uf nassem Wege. \\
$\mathrm{S}$ & 35,51 & $35,49 \%$ \\
$\mathrm{Zn}$ & 54,01 & $54,10 \%$ \\
$\mathrm{Fe}$ & 9,91 & $10,31 \%$ \\
$\mathrm{Gangart}$ & 0,21 & $0,12 \%$ \\
$\mathrm{~Pb}$ & Spuren & Spuren \\
\cline { 2 - 3 } & 99,64 & $100,02 \%$
\end{tabular}

\section{Analyse von Kupferkies.}

Die Einwirkung des Sauerstoffs erfolgt hier sehr leicht. das Selbsterglühen bereits nach zwei bis drei Minuten langer Einwirkung der Hitze, und in 10-15 Minuten ist alles Material in der Hauptsache oxydirt. Zum Schluss glüht man noch kräftig eine halbe bis dreiviertel Stunde in dem Sauerstoffstrome. Neben der Bildung der schwefligen Säure beobachtet man gleichzeitig ölige Tröpfchen, sowie reichliche Mengen von Schwefelsäuredampf, dessen vollständige Condensation man schliesslich zu beachten hat.

Resultate der Analyse des Kupferkieses von Neudorf am Harz.

0,5736 Grm. Substanz gaben 1,4325 Grm, BaSO $4=0,1969$ Grm. S; $0,2550 \mathrm{Grm}$. $\mathrm{Fe}_{2} \mathrm{O}_{3}=0,1785 \mathrm{Grm}$. Fe; $0,2492 \mathrm{Grm}$. $\mathrm{Cu}_{2} \mathrm{~S}=0,1989 \mathrm{Grm}$. Cu und $0,0006 \mathrm{Grm}$. Gangart, woraus sich die folgenden procentischen Verhältnisse berechnen.

\begin{tabular}{lc}
$\mathbf{S}$ & $34,33 \%$ \\
$\mathrm{Fe}$ & $31,12 \%$ \\
Cu & $34,68 \%$ \\
Gangart $\quad 0,10 \%$ \\
\hline
\end{tabular}


III. Analyse von Antimonit.

Der Antimonit oxydirt sich ebenfalls sehr leicht im Sauerstoffstrom, ähnlich dem Kupferkies. Leider verflüchtigt sich hierbei eine kleine Menge des entstandenen Antimonoxyds, so dass der Rückstand im Schiffchen nicht gewogen werden kann. Die milchig getrübte Schwefelsäurelösung wird vor ihrer Fällung filtrirt und mit einer geringen Menge Weinsäure versetzt, um darin vorhandene Spuren von Antimon bei der Fällung mit Baryumchlorid sicher in Lösung zu halten. Vielleicht gelingt es späteren Versuchen, die Verflüchtigung des Antimonoxyds auf irgend eine passende Art zu verhindern.

Resultate der Analyse reiner Antimonitkrystalle aus Japan.

1. 0,5702 Grm. Substanz, im Saucrstoffstrom oxydirt, gaben $=1,1831$ Grm. $\mathrm{BaSO}_{4}=0,1626 \mathrm{Grm}$. S.

2. Eine von Herrn Hefft nach meiner Methode ausgeführte Analyse. - 0,5721 Grm. Antimonit ergaben 1,1841 Grm. $\mathrm{BaSO}_{4}=0,1627 \mathrm{Grm}$. $\mathrm{S} ; 0,5715$ Grm. $\mathrm{Sb}_{2} \mathrm{~S}_{3}=0,4086$ Grm. Sb und 0,0007 Grm. Gangart.

3. Analyse des Antimonits auf nassem Wege (ebenfalls von Herrn Hefft unternommen). - 1,0388 Grm. Substanz lieferten 2,1460 Grm. $\mathrm{BaSO}_{4}=0,2950$ Grm. $s ; 1,0370$ Grm. $\mathrm{Sb}_{2} \mathrm{~s}_{3}=0,7414$ Grm. $\mathrm{Sb}$ und 0,0018 Grm. Gangart.

\begin{tabular}{lccc} 
& 1. & 2. & 3. \\
$\mathrm{Sb}$ & - & $\mathbf{7 1 , 4 2}$ & $\mathbf{7 1 , 3 7 \%}$ \\
$\mathrm{S}$ & $\mathbf{2 8 , 5 2}$ & 28,44 & $28,40 \%$ \\
Gangart & - & 0,12 & $0,17 \%$ \\
\cline { 3 - 4 } & & 99,98 & $99,94 \%$
\end{tabular}

Vou den Vortheilen der im Vorhergehenden beschriebenen Schwefel-Bestimmung in unorganischen Sulfiden gegenüber den gebräuchlichen Aufschliessungsmethoden auf nassem Wege wird sich wohl jeder Analytiker rasch überzeugen. Meine Methode gewährt nicht nur ein ungemein schnell zum Ziele führendes Arbeiten, sondern auch möglichste Genauigkeit, indem sie die für die Schwefelsäure so nachtheilige Gegenwart von Metallsalzen ausschliesst, welche nicht nur von dem Baryumsulfat in mehr oder weniger grösseren Mengen mitgerissen werden, sondern in der Wärme auch lösend auf den Niederschlag einwirken (wie $\mathrm{FeCl}_{3} ; \mathrm{CuCl}_{2} ; \mathrm{ZnCl}_{2}$ ). Nach meinen Erfahrungen wird bei der Schwefelsäure-Bestimmung ausser dem Eisen auch Mangan und Aluminium, unter Umständen auch Zink, Antimon, Kupfer etc. in bestimmten Quantitäten mitgefällt, Verhältnisse, welche bislang ganz genaue Analysen von eisen- 
haltiger Zinkblende, Kupferkies und verwandten Sulfiden sehr in die Länge zogen, wenn nicht geradezu unmöglich machten (vgl. beispielsweise die früheren Kupferkiesanalysen in Rammelsberg's Mineral-Analyse II. Aufl. 1875, S. 70).

Eine weitere Ausdehnung meiner Methode auf Sulfide wie Bleiglanz, Arsenkies, Molybdänsglanz u. s. f. behalte ich mir vor. Auch hoffe ich später das etwas unbequeme Bromwasser zweckmässiger durch eine concentrirtere Lösung von Wasserstoffsuperoxyd zu ersetzen, wenigstens haben einige vorläufige Versuche in dieser Richtung nicht ungünstige Resultate ergeben.

Heidelberg, Universitätslaboratorium, im April 1890.

\section{Z a r A bwe h r; \\ von}

F. Stohmann.

Im vorigen Hefte dieser Zeitschrift (Bd. 41, S. 424), sieht sich Herr Ossipoff, der in letzterer Zeit einige Bestimmungen des Wärmewerthes organischer Verbindungen ausgeführt hat, gemüssigt, einzelne der von mir und meinen Mitarbeitern veröffentlichten Zahlen zu bemängeln. Die Differenzen, um welche es sich in dem einen Fall handelt, sind so unbedeutender Art, dass es sich nicht dor Mühe lohnen würde, sie in Berücksichtigung zu ziehen. Wenn ich micb trotzdem zu einer Abwehr genöthigt sehe, so erfolgt diese, weil Herr Ossipoff dabei eine Aeusserung gethan hat, welche das ganze Wesen unserer Arbeitsweise berührt und geeignet ist, dieselbe in den Augen Mancher zu discreditiren.

Es kommt zunächst der Wärmewerth der Fumarsäure und der Maleïnsäure in Betracht. Für diese beiden Säuren sind folgende Zahlen gefunden:

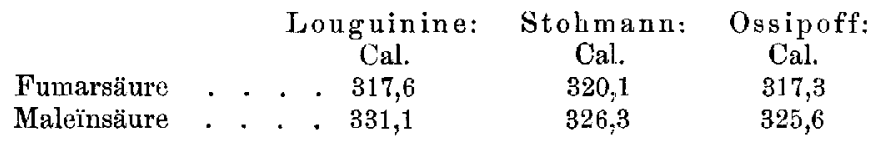

Hinsichtlich der Malë̈näure giebt Herr Ossip off unserer Zahl den Vorzug vor der von Louguinine, weil sie mit der seinigen übereinstimmt, glaubt aber, die für Fumarsäure von ihm und von Louguinine gefundene der unsrigen vorziehen zu sollen. 